Pesq. Vet. Bras. 35(11):875-881, novembro 2015 DOI: 10.1590/S0100-736X2015001100001

\title{
Efeitos de fontes orgânicas de cobre e enxofre sobre os parâmetros bioquímicos no soro de ovinos ${ }^{1}$
}

\author{
Renata M.C. Conti²*, Marcus A. Zanetti², Arlindo Saran Netto², Paulo H.M. Rodrigues ${ }^{3}$, \\ Jose C.G. Pacheco ${ }^{2}$, Carmen M.L.P. Garrine ${ }^{2}$ e Carolina Y.C. Yoshikawa ${ }^{2}$
}

\begin{abstract}
Conti R.M.C., Zanetti M.A., Saran Netto A., Rodrigues P.H.M., Pacheco J.C.G., Garrine C.M.L.P. \& Yoshikawa C.Y.C. 2015. [Effects of organic and inorganic copper and sulphur supplementation on blood biochemical parameters in sheep.] Efeitos de fontes orgânicas de cobre e enxofre sobre os parâmetros bioquímicos no soro de ovinos. Pesquisa Veterinária Brasileira 35(11):875-881. Departamento de Zootecnia, Faculdade de Zootecnia e Engenharia de Alimentos, Universidade de São Paulo, Av. Duque de Caxias Norte 225, Jardim Elite, Pirassununga, SP 13630-000, Brazil. E-mail: renataconti@usp.br

Minerals are essential components in the diet, exercising various functions in the animal. There are many studies to find better results for the organic sources of minerals, and one of the reasons for that is to avoid the negative interactions. The metabolic profile helps in assessment of production indices, therefore analyzes blood biochemical components. The objective of this study was to investigate the effects of organic and inorganic copper and sulphur supplementation on blood biochemical parameters in sheep. The experiment was conducted at the FZEA/USP. Total 40 weaned sheep were divided in a completely randomized design with 10 treatments: 1) basal diet; 2) basal diet plus Mo; 3) basal diet + inorganic Cu + inorganic S; 4) basal diet + inorganic $\mathrm{Cu}+$ organic S; 5) basal diet + organic $\mathrm{Cu}+$ inorganic $\mathrm{S}$; 6) basal diet + organic $\mathrm{Cu}+$ organic $\mathrm{S}$; 7) diet plus Mo + inorganic $\mathrm{Cu}+$ inorganic S; 8) diet plus Mo + inorganic $\mathrm{Cu}+\operatorname{organic} \mathrm{S}$; 9) diet plus Mo + organic $\mathrm{Cu}+$ inorganic S; 10) diet plus organic $\mathrm{Cu}+$ organic S. According each treatment was added $10 \mathrm{mg} \mathrm{kg}-1 \mathrm{MS}$ inorganic or organic $\mathrm{Cu}$ or $10 \mathrm{mg} \mathrm{kg-1} \mathrm{MS}$ Mo or $0.2 \% \mathrm{~S}$ inorganic or organic. The animals were fed twice a day, a total of $3 \%$ of body weight. The experiment lasted 84 days, blood was sampled every 28 days to study glucose, urea, albumin, cholesterol and triglycerides. The parameters were analyzed with factorial structure $2 \times 2 \times 2$ (with and without Mo, organic and inorganic $\mathrm{Cu}$ and organic and inorganic $S$ ) and a basal diet and a basal diet plus Mo, with 5\% significance. Serum levels of glucose, urea, albumin and cholesterol showed no significant difference between treatment and time ( $\mathrm{p}>0.05)$. It observed means of $64.8 \mathrm{mg} \mathrm{dL}^{-1} ; 30.0 \mathrm{mg} \mathrm{dl}^{-1} ; 2.78 \mathrm{mg}$ $\mathrm{L}^{-1} ; 72.2 \mathrm{mg} \mathrm{dL}^{-1}$ respectively for glucose, urea, albumin and cholesterol. Serum levels of triglycerides in the treatments $(28.8 ; 34.8 ; 30.8 ; 36.9 ; 34.3 ; 27.0 ; 31.6 ; 32.1 ; 34.6 ; 31.1 \mathrm{mg}$ $\mathrm{dL}^{-1}$ ) were influenced by interaction $\mathrm{Cu} \times \mathrm{S}$, triglycerides decreased with $\mathrm{Cu}$ and S organic.
\end{abstract}

INDEX TERMS: Copper, sulphur, mineral supplementation, glucose, albumin, cholesterol, triglyceride.

RESUMO.- Minerais são componentes essenciais na dieta, exercendo diversas funções no organismo animal onde o uso de sais orgânicos visa aumentar a disponibilidade dos

\footnotetext{
${ }^{1}$ Recebido em 29 de maio de 2015.

Aceito para publicação em 27 de outubro de 2015.

${ }^{2}$ Departamento de Zootecnia, Faculdade de Zootecnia e Engenharia de Alimentos, Universidade de São Paulo (USP), Av. Duque de Caxias Norte 225, Jardim Elite, Pirassununga, SP 13630-000, Brasil. *Autor para correspondência: renataconti@usp.br

${ }^{3}$ Departamento de Nutrição e Produção Animal, Faculdade de Medicina Veterinária e Zootecnia, USP, Av. Duque de Caxias Norte 225, Jardim Elite, Pirassununga, SP 13630-000.
}

minerais no trato digestório. 0 perfil metabólico auxilia na avaliação de índices produtivos, para tanto, se faz análises de componentes bioquímicos do sangue. 0 objetivo foi estudar os efeitos que fontes orgânicas e inorgânicas de cobre e enxofre possuem nos parâmetros bioquímicos. 0 experimento foi realizado na FZEA/USP, para tanto 40 ovinos foram distribuídos em 10tratamentos: 1) dieta basal; 2) dieta contendo Mo; 3) dieta basal+ $\mathrm{Cu}$ inorg + S inorg; 4) dieta basal $+\mathrm{Cu}$ inorg $+\mathrm{S}$ org; 5) dieta basal $+\mathrm{Cu}$ org + $\mathrm{S}$ inorg; 6) dieta basal $+\mathrm{Cu}$ org $+\mathrm{S}$ org; 7) dieta com Mo + $\mathrm{Cu}$ inorg $+\mathrm{S}$ inorg; 8) dieta com $\mathrm{Mo}+\mathrm{Cu}$ inorg $+\mathrm{S}$ org; 9) dieta com $\mathrm{Mo}+\mathrm{Cu}$ org $+\mathrm{S}$ inorg; 10) dieta com $\mathrm{Mo}+\mathrm{Cu}$ 
org + S org. De acordo com cada tratamento houve a inclusão de $10 \mathrm{mgkg}^{-1}$ de MS de Cu inorgânico ou orgânico ou 10 $\mathrm{mg} \mathrm{kg}^{-1}$ de MS de Mo ou 0,2\% de S inorgânico ou orgânico. Os animais receberam dieta única duas vezes ao dia com inclusão de volumoso num total de $3 \%$ do peso vivo. 0 experimento teve duração de 84 dias, com coletas a cada 28 dias para estudo de glicose, ureia, albumina, colesterol, triglicerídeos. Os parâmetros foram analisados com estrutura fatorial 2 × 2 × 2 (com e sem Mo, Cu orgânico e inorgânico e S orgânico e inorgânico) e uma dieta basal e uma basal mais molibdênio, em delineamento inteiramente casualizado, com um nível de significância de $5 \%$. Os teores séricos de glicose, ureia, albumina e colesterol não apresentaram diferença significativa entre tratamentos e tempo, tão pouco foram influenciados ( $p>0.05$ ) pelos tratamentos, sendo as médias $64,8 \mathrm{mg} \mathrm{dL}^{-1} ; 30,0 \mathrm{mg} \mathrm{dL}^{-1} ; 2,78 \mathrm{mg} \mathrm{L}^{-1} ; 72,2 \mathrm{mg} \mathrm{dL}^{-}$ ${ }^{-1}$ respectivamente, apresentando-se dentro da normalidade. Os teores de triglicerídeos nos tratamentos $(28.8 ; 34.8$; $30.8 ; 36.9 ; 34.3 ; 27.0 ; 31.6 ; 32.1 ; 34.6 ; 31.1 \mathrm{mg} \mathrm{dL}^{-1}$ ) foram influenciados pela interação Cu x S.

TERMOS DE INDEXAÇÃO: Cobre, enxofre, minerais, glicose, albumina, colesterol, triglicerideo.

\section{INTRODUÇÃO}

Os minerais são componentes essenciais na dieta de espécies domésticas (Baker et al. 2003), exercendo diversas funções no organismo, interagindo com outros nutrientes e entre si, tornando seu estudo complexo (Brizola \& Zanetti 1999).

A não suplementação mineral conforme as exigências nutricionais pode causar distúrbios metabólicos e com isso queda no desempenho produtivo (Berchielli et al. 2006).

Vários fatores podem alterar as respostas das suplementações minerais, como duração, forma e concentração do suplemento mineral, estado fisiológico do animal, fatores ambientais, estresse e minerais antagonistas (Baker et al. 2003, Malafaia et al. 2014).

A maior concentração de cobre no organismo se encontra no fígado, sua concentração aumenta com o crescimento e nível de produtividade. A absorção ocorre no trato intestinal, o transporte através do plasma e o armazenamento no fígado. A rota de excreção é a bílis (Cavalheiro \& Trindade 1992).

Em média trabalha-se com $10 \mathrm{mg} / \mathrm{kg}$ na ração, sendo necessário níveis maiores quando o teor de molibdênio é elevado. Para ovinos, a concentração máxima tolerada é de $15 \mathrm{mg} \mathrm{Cu} / \mathrm{kg}$ de MS na dieta, (NRC 2007), existindo proximidade entre os níveis desejados e toxidade, bem como uma dependência do nível de molibdênio (Berchielli et al. 2006), Ca, S, Fe e Zn (McDowell 2003).

No plasma encontra-se um complexo cobre-proteína denominado ceruloplasmina, globulina responsável pelo transporte de cobre no plasma e pela regulação da absorção e distribuição do mineral.

Segundo Gould \& Kendall (2011) a toxidade dos cobre-tiomolibdatos podem ser erroneamente confundidos com deficiência de cobre. A deficiência ou a toxidade de cobre depende da concentração de cobre na dieta e da concentra- ção de minerais antagonistas que interferem na sua absorção (Gooneratne et al. 1989), a quantidade de molibdênio na dieta pode ser aumentada para compensar os efeitos tóxicos de cobre em ovinos (Henry \& Miles 2000).

Na suplementação de enxofre utiliza-se flor de enxofre ou enxofre ventilado, sulfato de potássio ou sulfato de sódio como fonte de enxofre inorgânico (Cavalheiro \& Trindade 1992). Para ovinos, a concentração máxima tolerada $0,30 \%$ de $\mathrm{S} / \mathrm{kg}$ de MS em dietas com mais de $85 \%$ de concentrado (NRC 2007).

0 molibdênio é um mineral essencial, onde as maiores concentrações encontra-se no fígado e os rins. Sua absorção ocorre no trato intestinal e sua secreção é pela via urinária (Cavalheiro \& Trindade 1992).

0 molibdênio e o enxofre são antagonista ao cobres, ou seja, altos níveis de molibdênio aumentam as exigências de cobre. Caso os níveis de cobre no organismo sejam baixos, menores quantidades de molibdênio se tornarão tóxicas (Humphiries et al. 1983, Pott et al. 1999).

Sabe-se que aumento nos níveis de molibdênio no fígado, diminui a síntese de ceruloplasmina, tornando o cobre não disponível. 0 molibdênio em altos níveis interfere também no metabolismo energético.

Para ovinos, a concentração máxima tolerada é 05 a $10 \mathrm{mg}$ de Mo/kg de MS na alimentação quando considerado os níveis adequados de cobre (NRC 2007).

Os minerais inorgânicos são encontrados principalmente na forma de sulfatos, carbonatos, cloretos e óxidos, enquanto a forma orgânica geralmente são íons metálicos ligados a aminoácidos ou complexo de proteína (Baker 2003).

Segundo Baruselli (2008) os minerais orgânicos possuem maior absorção, maior retenção no organismo e, portanto, maior capacidade de promover os efeitos desejáveis da suplementação quando comparados com os minerais inorgânicos.

Várias interações entre minerais têm sido documentada, como zinco-ferro, cobre-enxofre, cobre-molibdênio, cobre-ferro, e cobre-molibdênio-enxofre (Baker 2003). Um excesso de consumo em um deles pode modificar o requerimento dos outros (Cavalheiro \& Trindade 1992).

Segundo Suttle (1975) existe possibilidade de que o enxofre orgânico tenha participação no efeito antagonista do cobre-molibdênio, porém, em experimento com enxofre orgânico e inorgânico observou que ambos tiveram papel similar no efeito antagonista do cobre- molibdênio- enxofre.

0 molibdato satura-se com as moléculas de sulfeto formando os mono, di, tri ou tetratiomolibdatos (Suttle 1991) que inibem o metabolismo do cobre, pois quanto maior a quantidade de enxofre na molécula, maior sua capacidade de ligar-se ao cobre livre (Suttle 1991) e na sequência ocorre a formação de cobre-tiomolibdatos que são altamente insolúveis (Vásquez et al. 2001) e indisponíveis para absorção (Galbraith et al 1997).

A interação cobre - molibdênio - enxofre produz efeito negativo sobre a utilização do cobre (Suttle 1975, Dick 1975), mesmo o enxofre ou molibdênio sozinhos podem ter efeito antagonista na absorção do cobre (Baker et al. 2003). 
Segundo Gonzáles (2000) o perfil metabólico auxilia na avaliação de índices produtivos, para tanto, se faz análises de componentes bioquímicos do sangue, como hematócrito, níveis de hemoglobina, glicose, ureia, albumina, globulina, proteína total, fosfatos inorgânicos, cálcio, potássio, magnésio, sódio, cobre e ferro.

Este trabalho teve como objetivo estudar os efeitos que as fontes orgânicas e inorgânicas de cobre e enxofre possuem nos parâmetros bioquímicos.

\section{MATERIAL E MÉTODOS}

O experimento foi realizado no Departamento de Zootecnia da Faculdade de Zootecnia e Engenharia de Alimentos da Universidade de São Paulo, sendo aprovado pelo Comitê de Ética em Pesquisa da Faculdade de Zootecnia e Engenharia de Alimentos sob o processo USP: 14.1.1410.74.7. Foram utilizados 40 ovinos inteiros, Santa Inês/Dorper, com idade aproximada de 3 meses e peso aproximado de $20 \mathrm{~kg}$ distribuídos em 10 tratamentos sendo 1) Dieta basal; 2) Dieta contendo 10mg de molibdênio; 3) Dieta basal + 10 mg de cobre inorgânico por kg de M.S. + 0,2\% de enxofre inorgânico; 4) Dieta basal $+10 \mathrm{mg}$ de Cu inorgânico + 0,2\% de enxofre orgânico; 5) Dieta basal + 10mg de Cu orgânico + 0,2\% de enxofre inorgânico; 6) Dieta basal $+10 \mathrm{mg}$ de Cu orgânico + 0,2\% de enxofre orgânico; 7) Dieta com 10mg de molibdênio + 10mg de cobre inorgânico + 0,2\% de enxofre inorgânico; 8) Dieta com 10mg de molibdênio + $10 \mathrm{mg}$ de cobre inorgânico + 0,2\% de enxofre orgânico; 9) Dieta com $10 \mathrm{mg}$ de molibdênio $+10 \mathrm{mg}$ de cobre orgânico $+0,2 \%$ de enxofre inorgânico; 10 ) Dieta com $10 \mathrm{mg}$ de molibdênio +10mg de cobre orgânico + 0,2\% de enxofre orgânico.

A fonte de enxofre inorgânico foi enxofre puro (flor de enxofre), o enxofre orgânico foi o enxofre-proteinato, o cobre inorgânico foi o sulfato de cobre e o cobre orgânico foi o cobre proteinado e a fonte de molibdênio utilizada foi o molibdato de sódio (Pott et al. 1999).

Antes do início do experimento, os animais receberam vermífugos e foram colhidas amostra de fígado através de biópsia cirúrgica.

Houve um período de adaptação de 14 dias antes do início da colheita das amostras, sendo que os animais receberam água e alimento à vontade até um máximo de $3 \%$ do peso vivo. Os animais permaneceram em gaiolas para estudo de metabolismo, revestidas de plástico para evitar possíveis contaminações, instaladas em galpão coberto equipado com cortinas laterais, comedouro e bebedouros individuais, durante um período experimental de 84 dias.

Os animais foram alimentados duas vezes ao dia através de ração total com inclusão de volumoso no intuito de evitar seleção (Zanetti \& Cunha 1997). As dietas foram formuladas para atender as exigências nutricionais recomendadas pelo NRC (2007), sendo que a dieta controle encontra-se no Quadro 1.

As análises bromatológicas foram realizadas nos Laboratórios de Bromatologia e de Minerais da Faculdade de Zootecnia e Engenharia de Alimentos da USP para análises de matéria seca (MS), proteína bruta $(\mathrm{PB})$, extrato etéreo (EE), matéria mineral (MM), energia bruta (EB) de acordo com AOAC (1996), fibra em detergente neutro (FDN) e fibra em detergente ácido (FDA), conforme Silva \& Queiroz (2005), cálcio, fósforo, cobre, molibdênio e enxofre foram determinadas por espectrometria de emissão ótica (ICP-OES) no laboratório de Minerais do Departamento de Zootecnia da FZEA/USP (Métodos Analíticos 2013).

Foram realizadas colheitas de sangue em cada animal por punção na veia jugular observando-se todos os cuidados de assepsia, a cada 28 dias, totalizando 84 dias, para as análises de glicose, colesterol, triglicerídeos, ureia e albumina e hematócrito.
Quadro 1. composição percentual da dieta experimental, na matéria original fornecida aos ovinos na dieta controle

\begin{tabular}{lc}
\hline \multicolumn{1}{c}{ Ingrediente } & Porcentagem \\
\hline Fubá de milho & 55,3 \\
Farelo de trigo & 1,0 \\
Casca de algodão & 25,0 \\
Farelo de soja & 12,5 \\
Óleo de soja & 1,0 \\
Calcário & 1,2 \\
Fosfato bicálcico & 2,0 \\
Ureia & 1,0 \\
Mistura vitamínica* & 0,5 \\
Mistura mineral ** & 0,5 \\
Total & 100,0
\end{tabular}

Fonte: Zanetti \& Cunha 1997. * Composição de vitaminas por quilograma de mistura mineral: Vit. A (400.000 UI) e Vit. E (4.000UI). ${ }^{* *}$ Composição dos minerais por quilograma de mistura mineral: I (100mg), Fe (4000mg), Co (40mg), Mn (3000mg), Se (40mg), Zn (4000mg) e NaCl (216g). Composição dos minerais experimentais: $\mathrm{Cu}, \mathrm{S}$, Mo.

Para as amostra de glicose e hematócrito foram utilizados tubos vaccuntainer com fluoreto na colheita de sangue. Para a determinação de hematócrito foi utilizado o método de micro hematócrito (Sink \& Feldman 2006). Os parâmetros sanguíneos ureia e albumina destinam-se ao metabolismo proteico e glicose, colesterol e triglicerídeos para metabolismo energético, sendo as análises determinadas pelo método enzimático.

Os parâmetros foram analisados considerando-se a existência de uma estrutura de tratamento fatorial $2 \times 2 \times 2$ (com e sem molibdênio, cobre orgânico e inorgânico, enxofre orgânico e inorgânico, além de uma dieta basal e uma basal com molibdênio), em delineamento inteiramente casualizado, com 4 repetições por tratamento e considerando cada animal como uma unidade experimental.

Para a comparação dos parâmetros nos diferentes tempos, os dados foram analisados utilizando-se o PROC MIXED do SAS (2000), com nível de significância de $5 \%$. Os contrates ortogonais estudados foram dieta basal x demais, Molibdênio exclusivo x demais, Presença de Molibdênio, Forma de Cobre, Forma de enxofre, Interação Molibdênio x Cobre, interação Molibdênio x enxofre, Interação cobre x enxofre e interação cobre x enxofre x molibdênio.

\section{RESULTADOS E DISCUSSÃO}

A análise bromatológica da dieta experimental apresentou 5,70\% MM; 4,28\% EE; 28,20 FDN; 16,64 FDA; 15,73\% PB; $1,18 \mathrm{Ca}$ e $0,58 \mathrm{P}$ na MS. Os teores de $\mathrm{Cu}$, Mo e $\mathrm{S}$ na dieta encontram-se no Quadro 2. Os resultados de glicose, albumina, ureia obtidos no período experimental estão apresentados nos Quadros 3, 4 e 5.

A concentração plasmática de glicose, albumina e ureia não apresentaram diferença significativa $(\mathrm{P}>0,05)$ entre os tratamentos, nem interação entre tempo*tratamento. Entretanto houve efeito quadrático em relação ao tempo para os parâmetros de glicose e ureia. A probabilidade estatística dos contrastes não mostraram efeito significativo para nenhum desses parâmetros.

Em estudo com sal orgânico e inorgânico Peixoto et.al. (2010) encontraram valores de 38mg/dl de ureia em média e $63 \mathrm{mg} / \mathrm{dl}$ de glicose, resultados estes semelhantes ao encontrado no presente estudo.

A concentração plasmática de glicose em ruminantes em jejum varia entre 77 a 115mg/dL (Kerr 2003). 
Quadro 2. Teores de cobre, molibdênio e enxofre nos tratamentos (em $\mathrm{mg} / \mathrm{kg})$

\begin{tabular}{cccc}
\hline Tratamento & Cobre & Molibdênio & Enxofre \\
\hline T0 & 9,96 & 1,02 & 2561,877 \\
T1 & 8,42 & 9,54 & 2369,516 \\
T2 & 15,7 & 1,05 & 4340,037 \\
T3 & 15,72 & 0,97 & 5178,186 \\
T4 & 17,22 & 1,18 & 4829,882 \\
T5 & 17,61 & 0,9 & 5209,693 \\
T6 & 15,74 & 11,6 & 4645,463 \\
T7 & 19,91 & 10,21 & 5288,507 \\
T8 & 16,67 & 11,92 & 5190,822 \\
T9 & 17,16 & 11,72 & 4906,294
\end{tabular}

A constância nas concentrações de glicose sanguínea se deve a eficiência de mecanismos hormonais como as vias glicolítica, gliconeogênese, glicogenólise e glicogênese (Berg et al. 2004).

As concentrações de albumina estão dentro da normalidade (Kerr 2003) e de acordo com valores encontrados por Ortolani (1997) em ovinos suplementados com molibdênio e enxofre e Peixoto et al. (2010) que suplementou ovinos com sal orgânico e inorgânico. Observa-se dessa maneira níveis adequados de proteína na dieta e ausência de efeitos tóxicos devidos aos tratamentos utilizados.

Níveis elevados de albumina podem ocorrer devido a desidratação, inflamações e níveis elevados ligados a gastroenterites, problemas hepáticos entre outros. (Sink \& Feldman 2006). Um fator importante para acompanhamento da albumina se deve ao fato dela e outras proteínas carreadoras se ligarem ao cobre no processo de absorção e carreá-lo ao fígado, local de estocagem para posterior utilização pelo organismo animal. A albumina se liga ao cobre transportando $10 \%$ do cobre plasmático, mas com menor

Quadro 3. Concentrações de glicose, em mg/dL, no plasma de ovinos recendo dieta controle ou suplementados com molibdênio ou fontes de cobre e enxofre

\begin{tabular}{|c|c|c|c|c|c|c|c|}
\hline \multicolumn{3}{|c|}{ Tratamentos } & \multicolumn{5}{|c|}{ Tempo (dias) } \\
\hline Mo & $\mathrm{Cu}$ & $S$ & 0 & 28 & 56 & 84 & Médi \\
\hline \multicolumn{8}{|c|}{ Combinações } \\
\hline Sem & Sem & Sem & 58,3 & 67,8 & 62 & 66,5 & 63,7 \\
\hline Com & Sem & Sem & 58,8 & 67,8 & 58,8 & 68,8 & 63,5 \\
\hline \multirow[t]{4}{*}{ Sem } & Inorgânico & Inorgânico & 54,3 & 65,5 & 60,7 & 61 & 60,3 \\
\hline & & Orgânico & 59,3 & 74,3 & 63,8 & 67,3 & 66,1 \\
\hline & Orgânico & Inorgânico & 61,5 & 70,5 & 62,7 & 70 & 66,4 \\
\hline & & Orgânico & 58 & 71,5 & 63,8 & 67,3 & 65,3 \\
\hline \multirow[t]{4}{*}{ Com } & Inorgânico & Inorgânico & 57,8 & 68 & 59,5 & 65 & 62,6 \\
\hline & & Orgânico & 61,3 & 72,8 & 63 & 68,5 & 66,4 \\
\hline & Orgânico & Inorgânico & 71,3 & 71,5 & 59 & 69,8 & 67,9 \\
\hline & & Orgânico & 64 & 69,8 & 62,3 & 70,8 & 66,7 \\
\hline \multicolumn{8}{|c|}{ Efeitos Principais } \\
\hline Sem & & & 58,4 & 70,4 & 62,9 & 66,4 & 64,6 \\
\hline Com & & & 63,6 & 70,5 & 60,9 & 68,5 & 65,9 \\
\hline & Inorgân & & 58,1 & 70,1 & 61,8 & 65,4 & 63,9 \\
\hline & Orgânico & & 63,8 & 70,8 & 61,9 & 69,4 & 66,6 \\
\hline & & Inorgânico & 61,2 & 68,9 & 60,3 & 66,4 & 64,3 \\
\hline & & Orgânico & 60,8 & 72,1 & 63,2 & 68,4 & 66,1 \\
\hline \multicolumn{8}{|c|}{ Dados Médios } \\
\hline Média & & & 60,4 & 69,8 & 61,6 & 67,4 & 64,8 \\
\hline EPM & & & 1,14 & 0,95 & 0,78 & 0,76 & 0,55 \\
\hline
\end{tabular}

Tratamento $=0,3180 ;$ Tempo $=0,0001 ;$ Tratamento ${ }^{*}$ Tempo $=0,3763 ;$ EPM = Erro padrão da média.
Quadro 4. Concentrações de albumina, em g/dL, no soro de ovinos recebendo dieta controle ou suplementados com molibdênio ou fontes de cobre e enxofre

\begin{tabular}{|c|c|c|c|c|c|c|c|}
\hline \multicolumn{3}{|c|}{ Tratamentos } & \multicolumn{5}{|c|}{ Tempo (dias) } \\
\hline Mo & $\mathrm{Cu}$ & $S$ & 0 & 28 & 56 & 84 & Méd \\
\hline \multicolumn{8}{|c|}{ Combinações } \\
\hline Sem & Sem & Sem & 2,61 & 2,64 & 2,67 & 2,69 & 2,65 \\
\hline Com & Sem & Sem & 2,96 & 2,86 & 2,92 & 2,84 & 2,85 \\
\hline \multirow[t]{4}{*}{ Sem } & Inorgânico & Inorgânico & 2,84 & 2,71 & 2,85 & 2,63 & 2,76 \\
\hline & & Orgânico & 2,76 & 2,81 & 2,86 & 2,91 & 2,83 \\
\hline & Orgânico & Inorgânico & 2,90 & 2,84 & 2,88 & 2,70 & 2,83 \\
\hline & & Orgânico & 2,80 & 2,71 & 2,80 & 2,86 & 2,79 \\
\hline \multirow[t]{4}{*}{ Com } & Inorgânico & Inorgânico & 2,86 & 2,91 & 2,90 & 2,80 & 2,87 \\
\hline & & Orgânico & 2,68 & 2,93 & 2,70 & 2,84 & 2,79 \\
\hline & Orgânico & Inorgânico & 2,59 & 2,69 & 2,81 & 2,71 & 2,70 \\
\hline & & Orgânico & 2,74 & 2,78 & 2,94 & 2,83 & 2,82 \\
\hline \multicolumn{8}{|c|}{ Efeitos Principais } \\
\hline Sem & & & 2,82 & 2,77 & 2,85 & 2,78 & 2,80 \\
\hline Com & & & 2,72 & 2,84 & 2,83 & 2,79 & 2,79 \\
\hline & Inorgânico & & 2,79 & 2,84 & 2,82 & 2,80 & 2,81 \\
\hline & Orgânico & & 2,76 & 2,76 & 2,86 & 2,78 & 2,79 \\
\hline & & Inorgânico & 2,80 & 2,8 & 2,86 & 2,71 & 2,79 \\
\hline & & Orgânico & 2,75 & 2,81 & 2,82 & 2,86 & 2,81 \\
\hline \multicolumn{8}{|c|}{ Dados Médios } \\
\hline Média & & & 2,75 & 2,78 & 2,82 & 2,78 & 2,78 \\
\hline EPM & & & 0,04 & 0,04 & 0,04 & 0,03 & 0,02 \\
\hline
\end{tabular}

Tratamento $=0,8242 ;$ Tempo $=0,3126$; Tratamento $*$ Tempo $=0,8314$; EPM = Erro padrão da média.

Quadro 5. Concentrações de uréia, em mg/dL, no soro de ovinos recebendo dieta controle ou suplementados com molibdênio ou fontes de cobre e enxofre

\begin{tabular}{|c|c|c|c|c|c|c|c|}
\hline \multicolumn{3}{|c|}{ Tratamentos } & \multicolumn{5}{|c|}{ Tempo (dias) } \\
\hline Mo & $\mathrm{Cu}$ & $S$ & 0 & 28 & 56 & 84 & Méd \\
\hline \multicolumn{8}{|c|}{ Combinações } \\
\hline Sem & Sem & Sem & 32,8 & 31,8 & 31,3 & 30,8 & 31,7 \\
\hline Com & Sem & Sem & 27,0 & 25,8 & 27,8 & 26,0 & 26,6 \\
\hline \multirow[t]{4}{*}{ Sem } & Inorgânico & Inorgânico & 32,8 & 25,8 & 24,8 & 22,5 & 26,4 \\
\hline & & Orgânico & 29,5 & 25,8 & 29,0 & 30,5 & 28,7 \\
\hline & Orgânico & Inorgânico & 34,3 & 31,3 & 31,0 & 35,5 & 33,0 \\
\hline & & Orgânico & 35,0 & 27,5 & 31,8 & 29,8 & 31,0 \\
\hline \multirow[t]{4}{*}{ Com } & Inorgânico & Inorgânico & 34,0 & 33,0 & 30,7 & 31,5 & 32,4 \\
\hline & & Org & 32,3 & 26,5 & 29,0 & 30,3 & 29,5 \\
\hline & Orgânico & Inorgânico & 32,5 & 31,3 & 29,3 & 29,0 & 30,5 \\
\hline & & Orgânico & 34,8 & 24,8 & 26,5 & 32,3 & 29,6 \\
\hline \multicolumn{8}{|c|}{ Efeitos Principais } \\
\hline Sem & & & 32,9 & 27,6 & 29,1 & 29,6 & 29,8 \\
\hline Com & & & 33,4 & 28,9 & 28,7 & 30,8 & 30,5 \\
\hline & Inorgân & & 32,1 & 27,8 & 28,2 & 28,7 & 29,2 \\
\hline & Orgânico & & 34,1 & 28,7 & 29,6 & 31,6 & 31,0 \\
\hline & & Inorgânico & 33,4 & 30,3 & 28,8 & 29,6 & 30,6 \\
\hline & & Orgânico & 32,9 & 26,1 & 29,1 & 30,7 & 29,7 \\
\hline \multicolumn{8}{|c|}{ Dados Médios } \\
\hline Média & & & 32,5 & 28,5 & 29,2 & 29,9 & 30,0 \\
\hline EPM & & & 0,88 & 0,87 & 0,89 & 0,95 & 0,46 \\
\hline
\end{tabular}

Tratamento $=0,2809 ;$ Tempo $=0,0004 ;$ Tratamento ${ }^{*}$ Tempo $=0,6913 ;$ EPM = Erro padrão da média.

afinidade que a ceruloplasmina, por isso a facilidade da albumina em liberar o cobre aos tecidos para estocagem.

A ureia é um metabólito envolvido na excreção de nitrogênio, como produto final da quebra de aminoácidos. Por ser um produto final na quebra de aminoácidos, a ureia pode sofrer alterações por fatores alimentares, como ex- 
Quadro 6. Concentrações de colesterol, em mg/dL, no soro de ovinos recebendo dieta controle ou suplementados com molibdênio ou fontes de cobre e enxofre

\begin{tabular}{|c|c|c|c|c|c|c|c|}
\hline \multicolumn{3}{|c|}{ Tratamentos } & \multicolumn{5}{|c|}{ Tempo (dias) } \\
\hline Mo & $\mathrm{Cu}$ & $S$ & 0 & 28 & 56 & 84 & Média \\
\hline \multicolumn{8}{|c|}{ Combinações } \\
\hline Sem & Sem & Sem & 65,3 & 70,8 & 60,2 & 64,8 & 65,3 \\
\hline Com & Sem & Sem & 70,5 & 67,5 & 69,5 & 65,5 & 68,3 \\
\hline \multirow[t]{4}{*}{ Sem } & Inorgânico & Inorgânico & 90,5 & 86,8 & 78,3 & 70,8 & 82,3 \\
\hline & & Orgânico & 69,5 & 75,5 & 63,8 & 67,3 & 69,0 \\
\hline & Orgânico & Inorgânico & $72, \varepsilon$ & 72,8 & 64,8 & 64,5 & 68,7 \\
\hline & & Orgâr & 77, & 76,8 & 67,5 & 74,5 & 73,9 \\
\hline \multirow[t]{4}{*}{ Com } & Inorgânico & Inorgânico & 71, & 83,0 & 72,0 & 71,3 & 74,5 \\
\hline & & Orgânic & 68,3 & 89,8 & 64,0 & 75,0 & 74,3 \\
\hline & Orgânico & Inorgânico & 64,3 & 86,3 & 79,8 & 73,3 & 75,9 \\
\hline & & Orgânico & 71,3 & 82,0 & 69,8 & 70,8 & 73,4 \\
\hline \multicolumn{8}{|c|}{ Efeitos principais } \\
\hline Sem & & & 78,2 & 77,9 & 68,6 & 69,3 & 73,5 \\
\hline Com & & & 68,7 & 85,3 & 71,3 & 72,6 & 74,5 \\
\hline & Inorgân & & 75,6 & 83,7 & 69,3 & 71,1 & 75,0 \\
\hline & Orgânico & & 71,3 & 79,4 & 70,4 & 70,8 & 73,0 \\
\hline & & Inorgânico & 75,4 & 82,2 & 73,8 & 69,9 & 75,3 \\
\hline & & Orgânico & 71,5 & 81,0 & 66,3 & 71,9 & 72,7 \\
\hline \multicolumn{8}{|c|}{ Dados médi } \\
\hline Média & & & 72,0 & 78,7 & 68,4 & 69,5 & 72,2 \\
\hline EPM & & & 2,42 & 2,22 & 1,94 & 2,13 & 1,13 \\
\hline
\end{tabular}

Tratamento $=0,4646 ;$ Tempo $=0,0001 ;$ Tratamento $*$ Tempo $=0,3767$; $\mathrm{EPM}=$ Erro padrão da média.

Quadro 7. Concentrações de triglicerídeos, em mg/dL, no soro de ovinos recebendo dieta controle ou suplementados com molibdênio ou fontes de cobre e enxofre

\begin{tabular}{|c|c|c|c|c|c|c|c|}
\hline \multicolumn{3}{|c|}{ Tratamentos } & \multicolumn{5}{|c|}{ Tempo (dias) } \\
\hline Mo & $\mathrm{Cu}$ & $\mathrm{S}$ & 0 & 28 & 56 & 84 & Média \\
\hline \multicolumn{8}{|c|}{ Combinações } \\
\hline Sem & Sem & Sem & 32,7 & 27,5 & 27,3 & 27,7 & 28,8 \\
\hline Com & Sem & Sem & 35,5 & 34,8 & 42,5 & 26,5 & 34,8 \\
\hline \multirow[t]{4}{*}{ Sem } & Inorgânico & Inorgânico & 32,8 & 32,5 & 36,3 & 21,8 & 30,8 \\
\hline & & Orgânico & 41,5 & 33,0 & 36,8 & 36,3 & 36,9 \\
\hline & Orgânico & Inorgânico & 40,8 & 32,5 & 36,0 & 27,8 & 34,3 \\
\hline & & Orgânico & 26,3 & 24,3 & 29,8 & 27,8 & 27,0 \\
\hline \multirow[t]{4}{*}{ Com } & Inorgânico & Inorgânico & 32,0 & 27,3 & 34,5 & 32,5 & 31,6 \\
\hline & & Orgânico & 34,0 & 33,5 & 28,0 & 32,8 & 32,1 \\
\hline & Orgânico & Inorgânico & 31,5 & 38,0 & 38,3 & 30,8 & 34,6 \\
\hline & & Orgânico & 29,8 & 30,8 & 34,3 & 29,5 & 31,1 \\
\hline \multicolumn{8}{|c|}{ Efeitos principais } \\
\hline Sem & & & 35,3 & 30,6 & 34,7 & 28,4 & 32,2 \\
\hline Com & & & 31,8 & 30,4 & 33,8 & 31,4 & 32,3 \\
\hline & Inorgâr & & 35,1 & 31,6 & 33,9 & 30,8 & 32,8 \\
\hline & Orgânico & & 32,1 & 31,4 & 34,6 & 28,9 & 31,7 \\
\hline & & Inorgânico & 34,3 & 32,6 & 36,3 & 28,2 & 32,8 \\
\hline & & Orgânico & 32,9 & 30,4 & 32,2 & 31,6 & 31,8 \\
\hline \multicolumn{8}{|c|}{ Dados médios } \\
\hline Média & & & 33,6 & 31,2 & 34,0 & 29,2 & 32,0 \\
\hline EPM & & & 1,27 & 1,30 & 1,25 & 0,98 & 0,61 \\
\hline
\end{tabular}

Tratamento $=0,1456 ;$ Tempo $=0,0026$; Tratamento ${ }^{*}$ Tempo $=0,1690$; $\mathrm{EPM}=$ Erro padrão da média.

cesso na alimentação, ou proteína de baixa qualidade, deficiência de carboidrato entre outros (Kerr 2003)auxiliando assim a avaliação alimentar e o desenvolvimento da saúde animal. Portanto, fatores que aumentam a produção de ureia no fígado, promovem elevação na concentração sanguínea de ureia (Thrall et al. 2006). A concentração plas- mática normal de ureia em animais é de 3 a $8 \mathrm{mmol} / \mathrm{L}$ (Kerr 2003).

Nos Quadros 6 e 7 encontram-se os valores séricos de colesterol e triglicerídeos.

Não foram observadas diferenças significativas $(\mathrm{P}>0,05)$ entre os tratamentos para os parâmetros de colesterol total e triglicerídeos no soro dos ovinos. Observou-se efeito quadrático em relação ao tempo $(\mathrm{P}<0,05)$ para os parâmetros de colesterol e triglicerídeos no soro. A interação cobre-enxofre mostrou efeito significativo $(\mathrm{P}<0,05)$ nos níveis de triglicerídeos, diminuindo os valores sanguíneos de triglicerídeos quando administrado cobre e enxofre nas fontes orgânicas, como pode ser observado também na Figura 1.

Em relação ao colesterol, não foi observado diferença significativa entre os tratamentos e a probabilidade estatística dos contrastes também não apresentou efeito significativo $(\mathrm{P}>0,05)$. Ocorreu efeito no tempo, sendo que na presença de molibdênio ocorreu aumento nos níveis de colesterol e redução nos tratamentos sem a inclusão de molibdênio.

Os triglicerídeos não apresentaram efeito significativo em relação aos tratamentos $(\mathrm{P}>0,05)$. A interação cobre $\mathrm{x}$ enxofre mostrou efeito significativo $(P<0,05)$ sendo que na presença de cobre e enxofre nas fontes orgânicas houve redução nos níveis de triglicerídeos conforme apresentado na Figura 1.

O conteúdo dos triglicerídeos na corrente sanguínea em níveis normais reflete o equilíbrio entre absorção no intestino delgado, sua síntese e secreção nos hepatócitos e absorção pelo tecido adiposo (Thrall et al. 2006). Os valores médios encontrados neste estudo estão de acordo com os apresentados por Peixoto et al. (2010), sendo $73 \mathrm{mg} / \mathrm{dL}$ para colesterol e $23 \mathrm{mg} / \mathrm{dL}$ de triglicerídeos plasmáticos.

Garrine (2013) não observou efeito significativo $(\mathrm{P}>0,05)$ nas concentrações de triglicerídeos e colesterol total plasmático com suplementação de $\mathrm{Cu}$ ficando os valores de colesterol total entre 82 e $96 \mathrm{mg} / \mathrm{dl}$ e os valores de triglicerídeos entre 19 e $27 \mathrm{mg} / \mathrm{l}$.

Em outro estudo com adição de 10 ou $20 \mathrm{mg}$ cobre $/ \mathrm{kg}$ MS (cobre-lisina e cloreto do cobre tribásico) na alimentação de ovinos, Cheng et al. (2008) observaram redução nos níveis de colesterol sanguíneo quando adicionado cobre, independente da fonte e aumento nas concentrações de triglicerídeos.

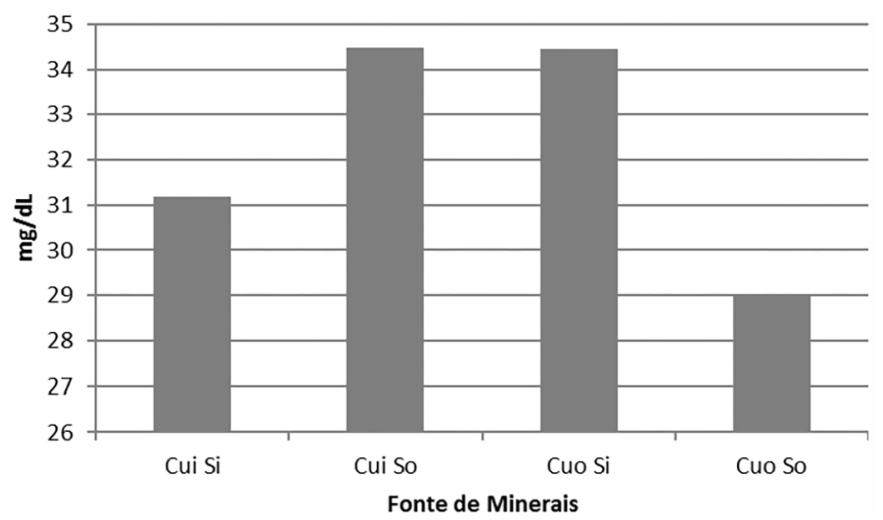

Fig.1. Níveis médios de triglicerídeos no soro de ovinos na interação de fontes orgânicas e inorgânicas de cobre e enxofre. 
Quadro 8. Concentrações de hematócrito, em porcentagem, no soro de ovinos recebendo dieta controle ou suplementados com molibdênio ou fontes de cobre e enxofre

\begin{tabular}{|c|c|c|c|c|c|c|c|}
\hline \multicolumn{3}{|c|}{ Tratamentos } & \multicolumn{5}{|c|}{ Tempo (dias) } \\
\hline Mo & $\mathrm{Cu}$ & $S$ & 0 & 28 & 56 & 84 & Média \\
\hline \multicolumn{8}{|c|}{ Combinações } \\
\hline Sem & Sem & Sem & 28,83 & 29,17 & 28,17 & 28,25 & 28,60 \\
\hline Com & Sem & Sem & 32,25 & 30,88 & 31,25 & 27,88 & 30,56 \\
\hline \multirow[t]{4}{*}{ Sem } & Inorgânico & Inorgânico & 32,75 & 33,88 & 33,13 & 32,38 & 33,03 \\
\hline & & Orgânico & 31,63 & 33,25 & 31,38 & 30,13 & 31,59 \\
\hline & Orgânico & Inorgânico & 33,50 & 32,88 & 31,88 & 30,75 & 32,25 \\
\hline & & Orgâ & 31,88 & 30,88 & 29,50 & 29,63 & 30,47 \\
\hline \multirow[t]{4}{*}{ Com } & Inorgânico & Inorgânico & 32,00 & 31,88 & 31,75 & 30,88 & 31,63 \\
\hline & & Org & 29,75 & 31,00 & 30,00 & 27,38 & 29,53 \\
\hline & Orgânico & Inorgânico & 28,00 & 29,25 & 28,75 & 29,50 & 28,88 \\
\hline & & Orgânico & 32,00 & 33,38 & 29,38 & 27,00 & 30,19 \\
\hline \multicolumn{8}{|c|}{ Efeitos principais } \\
\hline Sem & & & 32,44 & 32,72 & 31,47 & 30,72 & 31,84 \\
\hline Con & & & 30, & 31,13 & 29,97 & 28,69 & 30,05 \\
\hline & Inorg & & 31,5 & 32,50 & 31,56 & 30,19 & 31,45 \\
\hline & Orgân & & 31,34 & 31,34 & 29,88 & 29,22 & 30,45 \\
\hline & & Inorgânico & 31,56 & 31,97 & 31,38 & 30,88 & 31,45 \\
\hline & & Orgânico & 31,31 & 31,88 & 30,06 & 28,53 & 30,45 \\
\hline \multicolumn{8}{|c|}{ Dados médios } \\
\hline Méd & & & 31,14 & 31,43 & 30,40 & 29,32 & 30,57 \\
\hline EPM & & & 0,52 & 0,47 & 0,52 & 0,45 & 0,25 \\
\hline
\end{tabular}

Probabilidades estatísticas dos contrates

Tratamento $=0,1932 ;$ Tempo $=0,0001 ;$ Tratamento ${ }^{*}$ Tempo $=0,6192 ;$ $\mathrm{EPM}=$ Erro padrão da média

Segundo Bakalli (1995) a síntese de colesterol pode ter relação com a suplementação de cobre, pois o aumento do cobre hepático pode diminuir a concentração de glutationa reduzida e consequentemente reduzir a enzima limitante na síntese e colesterol, denominada 3-hidroxi-3 metil glutaril coenzima A-redutase (HMG-CoA redutase).

Os valores de hematócrito aparecem no Quadro 8, não apresentaram diferença significativa entre os tratamentos $(P>0,05)$ independente da inclusão de minerais ou das fontes, somente efeito quadrático no tempo.

Os valores de hematócrito em ovinos encontrados neste estudo estão dentro da normalidade, que pode variar de 27 a 45\% (Garcia-Navarro \& Pachaly 1994) e a normalidade também indica que não houve problema por excesso de cobre.

\section{CONCLUSÃO}

A suplementação com molibdênio e/ou fontes de cobre e enxofre reduziram as concentrações de triglicerídeos na presença de fontes orgânicas de cobre e enxofre; porém não foram observados efeitos da interação cobre-enxofre-molibdênio nos outros parâmetros bioquímicos estudados.

Agradecimentos.- Ao Fundo de Amparo a Pesquisa do Estado de São Paulo (FAPESP) pelo financiamento do referido projeto (Projeto FAPESP 2011/21377-0). Á Coordenação de Aperfeiçoamento de Pessoal de Nível Superior (CAPES), e ao Conselho Nacional de Desenvolvimento Científico e Tecnológico (CNPq) pela concessão de bolsa de Doutorado.

\section{REFERÊNCIAS}

Association of Official Analytical Chemists 1996. Official Methods of Analysis.16th ed. AOAC, Arlington. 1298p.
Bakalli R.I., Pesti G.M., Ragland W.L. \& Konjufca V. 1995. Dietary copper in excess of nutritional requirement reduces plasma and breast muscle cholesterol of chickens. Poult. Sci. 74(2):360-365.

Baker D.S., Ahola J.K., Burns P.D. \& Engle T.E. 2003. Trace mineral metabolism in ruminants: impact in production, reproduction and the environment. Proc. Alltech's 19th Annual Symposium on Nutritional Biotechnology in the Feed and Food Industries., Fort Collins, Colorado, p.275-287.

Baruselli M.S. 2008. Benefícios do uso de minerais sob a forma orgânica no balanceamento de rações para ruminantes. Anais 1 ํㅡㄹ Congresso Brasileiro de Nutrição Animal, Fortaleza, p.1-21.

Berchielli T.T., Pires A.V. \& Oliveira S.G. 2006. Nutrição de Ruminantes. Funep, Jaboticabal 583p.

Berg J.M., Tymoczko J.L. \& Strayer L. 2004. Bioquímica. 5aㅡ ed. Guanabara Koogan, Rio de Janeiro. 1059p.

Brizola M.L. \& Zanetti M.A. 1999. Efeitos de níveis elevados de selênio e enxofre na dieta de cordeiros sobre o metabolismo do cobre, do zinco e do ferro. Anais XXXVI Reunião Anual da Sociedade Brasileira de Zootecnia, Porto Alegre. Disponível em <http://www.sbz.org.br/visualizar. php?idiom=pt\&artigo=17917> Acesso em 8 out. 2014.

Cavalheiro A.C.L. \& Trindade D.S. 1992. Os Minerais para Bovinos e Ovinos Criados em Pastejo. Sagra, Porto Alegre. 142p.

Cheng J., Fan C., Zhang W., Zhu X., Yan X., Wang R. \& Jia Z. 2008. Effects of dietary copper source and level on performance carcass characteristics an lipid metabolism in lambs. Asian-Aust. J. Anim. Sci. 21(5):685-691.

Dick A.T., Dewwy D.W. \& Gawthorne J.M. 1975. Thiomolybdates and copper-molybdenum-sulfur interaction in ruminant nutrition. J. Agric. Sci. 85:567-568.

Galbraith H., Chigwada W., Scaife J.R. \& Humphries W.R. 1997. The effect of dietary molybdenum supplementation on tissue copper concentrations, mohair fibre and carcass characteristics of growing Angora goats. Anim. Feed Sci. Technol. 67:83-90.

Garcia-Navarro C.E.K. \& Pachaly J.R. 1994. Técnicas hematológicas, p.6989. In: Ibid. (Eds), Manual de Hematologia Veterinária. Livraria Varela, São Paulo.

Garrine C.M.L.P. 2013. Efeito de dois níveis de sulfato e cobre e cobre metionina no metabolismo e oxidação de lipídeos em ovinos. Tese de Doutorado em Zootecnia, Faculdade de Zootecnia e Engenharia de Alimentos, Universidade de São Paulo, Pirassununga, SP. 94p.

Gould L. \& Kendall N.R. 2011. Role of the rumen in copper and thiomolybdate absorption. Nutr. Res. Revs 24:176-182.

González F.H.D., Durr J.W. \& Fontaneli R.S. 2000. Perfil Metabólico em Ruminantes: seu uso em nutrição e doenças nutricionais. Gráfica UFRGS, Porto Alegre.

Gooneratne S.R., Buckley W.T. \& Christensen D.A. 1989. Review of cooper deficiency and metabolism in ruminants. Can. J. Anim. Sci. 679:819-845.

Henry P.R. \& Miles R.D. 2000. Interactions among the trace minerals. Ciênc. Anim. Bras.1:95-106.

Humphries W.R., Phillippo M., Young B.W. \& Bremner I. 1983. The influence of dietary iron and molybdenum on copper metabolism in calves. Brit. J. Nutr. 49(1):77-86.

Kerr M.G. 2003. Introdução à bioquímica clínica, p.81-230. In: ibid. (Ed.), Exames Laboratoriais em Medicina Veterinária, Bioquímica Clínica e Hematologia. $2^{\text {a }}$ ed. Editora Roca, São Paulo.

Malafaia P., Costa R.M., Brito M.F., Peixoto P.V., Barbosa J.D., Tokarnia C.H. \& Döbereiner J. 2014. Equívocos arraigados no meio pecuário sobre deficiências e suplementação minerais em bovinos no Brasil. Pesq. Vet. Bras. $34: 244-249$

McDowell L.R. 2003. Minerals in Animal and Human Nutrition. 2nd ed. Elsevier, Amsterdam, Netherlands. 644p.

Métodos Analíticos 2013. Compêndio Brasileiro de Alimentação Animal. 4 a ed. Sindirações, São Paulo, p.175-181.

NRC 2007. Nutrient Requeriments of Small Ruminants. National Research Council, Washington, DC. 362p.

Ortolani E.L. 1997. Efeitos da suplementação dieta de molibdênio e enxofre 
sobre a infestação de Haemonchus contortus (Rudolphi, 1803) em ovinos: estudo de alguns aspectos do metabolismo de cobre e sódio e da resposta celular do hospedeiro. Tese de Livre-Docência, Faculdade de Medicina Veterinária e Zootecnia, Universidade de São Paulo, São Paulo. 94p.

Peixoto L.A.O., Osorório M.T.M., Osório J.C.S., Nornberg J.L. \& Pazini M. 2010. Desempenho reprodutivo em metabólitos sanguíneos de ovelhas Île de France sob suplementação com sal orgânico ou sal comum durante estação de monta. Revta Bras. Zootec. 39(1):191-197.

Pott E.B., Henry P.R., Zanetti M.A., Rao P.V., Hinderberger Jr E.J. \& Ammerman C.B. 1999. Effects of high dietary molybdenum concentration and duration of feeding time on molybdenum and copper metabolism in sheep. Anim. Feed Sci. Technol. 79(1/2):93-105.

SAS 2000. Guide for Personal Computers. SAS Institute Inc., Cary.

Silva D.J. \& Queiroz A.C. 2005. Análise de Alimentos: métodos químicos e biológicos. 3ํe ed. Editora UFV, Viçosa, MG. 235p.
Sink C.A. \& Feldman B.F. 2006. Hematologia: coleta e avaliação da amostra, p.45-60. In: Ibid. (Eds), Urinálise e Hematologia: laboratorial para o clínico de pequenos animais. Editora Roca, São Paulo.

Suttle N.F. 1975. The role of organic sulphur in the copper-molybdenum-S interrelationship in ruminant nutrition. Brit. J. Nutr. 34:411-420.

Suttle N.F. 1991. The interaction between copper, molybdenum and sulfur in ruminants nutrition. Annual Vet. Nutr. 11:121-140.

Thrall M.A., Baber D.C., Campbell T.W., Denicola D., Fettman M.J., Lassen E.D., Rebar A. \& Weise G. 2006. Bioquímica clínica aplicada aos principais animais domésticos, p.282-430. In: Ibid. (Eds), Hematologia e Bioquímica Clínica Veterinária. Editora Roca, São Paulo.

Vásquez E.F.A., Herrera A.P.N. \& Santiago G.S. 2001. Interação cobre, molibdênio e enxofre em ruminantes. Ciência Rural 31(6):1101-1106.

Zanetti M.A. \& Cunha J. 1997. Biodisponibilidade de fontes orgânicas e inorgânicas de selênio. Revta Bras. Zootec. 26(3):623-627. 